\title{
Explorando correlações em Programação: um estudo focado no processo seletivo e em disciplinas correlatas
}

\author{
Marlos Tacio Silva ${ }^{1}$, Maria Cristina Tenório Cabral Cavalcante ${ }^{2}$, \\ Evandro de Barros Costa ${ }^{2}$
}

${ }^{1}$ Departamento de Sistemas e Computação - Universidade Federal de Campina Grande Campina Grande - PB - Brasil

\author{
${ }^{2}$ Instituto de Computação - Universidade Federal de Alagoas \\ Maceió - AL - Brasil \\ marlos@copin.ufcg.edu.br, cristina.tenorio@gmail.com, evandro@ic.ufal.br
}

\begin{abstract}
This paper presents a study produced with data of entering students of a Computer Science course at Federal University of Alagoas in the years 2011 and 2012 (respectively, 47 and 68 students), which aims to identify correlations between the discipline of Programming 1 and the performance obtained in both the selection process and in the related disciplines. We can highlight the following results: 1) a moderate correlation between Programming and Programming Laboratory ( $\rho=0.61, p$-value $<0.001), 2)$ a moderate negative correlation between Programming and Humanities and Languages (respectively, $\rho=-0.38, p-$ value $<0.010$, and $\rho=0.28, p-$ value $<0.100)$, and 3) a very weak correlation between Programming and disciplines of Math.
\end{abstract}

\begin{abstract}
Resumo. O presente trabalho apresenta um estudo realizado com dados de turmas de Ciência da Computação da Universidade Federal de Alagoas ingressantes nos anos de 2011 e 2012 (respectivamente, 47 e 68 estudantes) que visa identificar correlações entre a disciplina de Programação 1 e o desempenho obtido tanto no processo seletivo quanto em disciplinas correlatas. Dentre os resultados obtidos podem-se destacar: 1) um coeficiente de correlação moderado entre Programação e Laboratório de Programação $(\rho=0,61, p-$ valor $<0.001)$; 2) uma correlação negativa entre Programação e o desempenho em Humanas e Linguagens (respectivamente, $\rho=-0.38$, $p-$ valor $<0.010$; e $\rho=0.28$, $p$ - valor < 0.100); e 3) correlações muito fracas entre Programação e disciplinas de Matemática.
\end{abstract}

\section{Introdução}

Tradicionalmente, o ensino de programação é tido como uma atividade onerosa, sobretudo para turmas introdutórias, sendo considerado um dos grandes desafios da Informática na Educação [McGettrick et al. 2005]. Sob essa perspectiva, um estudo realizado por Bennedsen e Caspersen [Bennedsen and Caspersen 2007] com 63 instituições de 15 países apresenta taxas de retenção de aproximadamente $30 \%$, considerado um índice alarmante principalmente se for levada em consideração a importância dessa disciplina no decorrer de um curso de Computação. 
Nesse contexto, diversos problemas e, consequentemente, soluções tem sido apontados como efetivas para o ensino de programação. Dentre os problemas levantados pode-se citar, por exemplo, a carência de ferramentas computacionais [Powers et al. 2006], a organização curricular [Pears et al. 2007], a ineficácia do processo avaliativo [de Jesus and Raabe 2009], a baixa capacidade de abstração [Piva Jr and Freitas 2010], entre outros.

Assim, é possível notar que existe uma série de fatores que estão sendo utilizados no intuito de explicar o problema do ensino de programação. Entretanto, tais fatores precisam ser melhor estudados e avaliados. Em outras palavras, é necessário compreender melhor como os diversos fatores influenciam de fato o desempenho de um estudante de programação. Nesse sentido, diversos autores têm realizado estudos visando compreender melhor o fenômeno do ensino de programação e os fatores que podem afetar o mesmo (e.g., [Bergin and Reilly 2005], [Lahtinen et al. 2005], [Carbone et al. 2009] e [Hertz and Ford 2013]).

Nesse sentido, a proposta deste trabalho é a de estudar um conjunto de fatores no intuito de verificar possíveis correlações com o desempenho em disciplinas introdutórias de programação, doravante denominada Programação 1. Para tal, será realizada uma análise em dados históricos de turmas ingressantes nos anos de 2011 e 2012 do curso de Ciência da Computação da Universidade Federal de Alagoas. Nesse sentido, visa-se, de modo geral, analisar o coeficiente de correlação entre a disciplina de Programação 1 e o desempenho obtido no processo seletivo (i.e., as notas do ENEM em cada uma de suas áreas de conhecimento), bem como o desempenho obtido em disciplinas correlatas do curso (i.e., as demais disciplinas do primeiro módulo do curso).

Os resultados obtidos mostram que as disciplinas do curso apresentam correlações mais fortes do que o desempenho obtido no processo seletivo. Além disso, o desempenho em matemática se mostrou fracamente correlacionado ao desempenho de Programação 1, resultado considerado inesperado, haja vista a capacidade de raciocínio necessária para programar. Frisa-se que este estudo é inicial e, como tal, necessita de mais dados para que os resultados possam ser generalizados.

\section{Trabalhos Relacionados}

A presente seção tem o objetivo de apresentar os trabalhos relacionados ao estudo em questão. Para tal buscou-se focalizar trabalhos com base nos critérios a seguir: 1) publicados em conferências com Qualis maior ou igual a B2; 2) foco no ensino introdutório de programação; 3) foco no estudo de fatores que influenciam o desempenho do estudante; 4) apresentam algum tipo de experimento que valide minimamente seus resultados.

O trabalho de [Bergin and Reilly 2005] argumenta que as disciplinas introdutórias de programação tendem a ter um número muito grande de estudantes e os professores não conseguem saber o quão bem os estudantes estão indo antes da primeira avaliação. Tal avaliação pode ser muito tarde para aplicar alguma intervenção que possa prevenir no restante da disciplina. Além disso, mesmo após a primeira avalição, o professor tem poucas informações que possam ajudá-lo a identificar problemas individuais e, por conseguinte, aplicar a intervenção mais adequada. Nesse sentido, o trabalho apresenta um estudo que visa o levantamento de fatores que podem influenciar o desempenho de estudantes em disciplinas introdutórias de programação. O estudo foi realizado com estudantes de uma 
turma introdutória de programação visando coletar informações sobre as seguintes categorias: 1) experiência prévia, que foram obtidas pelo desempenho no Irish Leaving Certificate; 2) habilidades cognitivas específicas, mensuradas por um teste cognitivo realizado no curso; 3 ) informações pessoais; e 4) percepção pessoal do curso, ou seja, quão confortáveis os estudantes se sentem com o material do curso. Os dados foram coletados a partir de duas fontes distintas: via questionário; e via um teste cognitivo.

O trabalho de [Lahtinen et al. 2005] argumenta que os cursos introdutórios de programação geralmente apresentam dificuldades no processo de ensino e, sendo assim, tais dificuldades devem ser reconhecidas no intuito de auxiliar o ensino de forma efetiva. Assim, o trabalho apresenta um questionário aplicado com mais de 500 estudantes e professores que visa prover informações sobre as dificuldades encontradas no ensino e aprendizado de programação. O trabalho apresenta um questionário composto por três seções: 1) background; 2) conteúdo do curso; e 3) aspectos de aprendizagem. Nesse sentido, esse questionário foi aplicado tanto para estudantes, visando mensurar as dificuldades encontradas por eles no decorrer do curso, quanto para professores, no intuito de mensurar a opinião do professor com relação à dificuldades do curso. Assim, o objetivo é o de comparar as diferentes concepções entre professores e estudantes. O estudo envolveu um total de 559 estudante e 34 professores distribuídos em seis universidades de diferentes países da Europa (i.e., Alemanha, Islândia, Finlândia, Romênia e Látvia).

O trabalho de [Carbone et al. 2009] argumenta que existem muitos fatores que podem influenciar o processo de ensino de Programação 1. Entre estes pode-se listar fatores individuais ou internos, fatores ambientais ou externos. Nesse sentido, o trabalho realiza um estudo acerca dos fatores internos que podem influenciar o processo de ensino sob a perspectiva de duas dimensões: (1) motivação; e (2) capacidade. O estudo envolveu estudantes do primeiro ano dos cursos de Ciência da Computação e Sistema de Informação da Monash University uma universidade da Austrália. O estudo envolveu a coleta de dados de duas fontes distintas: 1) entrevistas semiestruturadas; e 2) relatos escritos de estudantes envolvidos na realização de tarefas. A partir da análise dos resultados obteve-se um arcabouço conceitual que estrutura os fatores internos.

O trabalho de [Hertz and Ford 2013] argumenta que um professor de programação geralmente lida com uma série de decisões durante o projeto e a execução de um curso de programação (e.g., quais tópicos abordar e quanto tempo dedicar a cada um desses tópicos). Nesse sentido, tais decisões podem influenciar positivamente ou negativamente no desempenho da turma. Assim o trabalho visa caracterizar a correlação entre os tópicos do curso e o tempo ou a importância dada a este tópico. Para tal, foi aplicado um survey com 58 professores no intuito de mapear a importância dada a cada tópico e o desempenho dos estudantes nos respectivos tópicos.

\section{Metodologia}

Nesta seção serão apresentados os objetivos do estudo em questão, bem como o projeto e a execução do mesmo. Além disso, serão apresentados alguns detalhes acerca da seleção dos valores observados e dos instrumentos utilizados na análise dos dados.

\subsection{Objetivos do Estudo}

De modo sucinto, o presente estudo visa realizar uma investigação acerca de fatores que apresentam alguma relação com o desempenho Programação 1. Mais especificamente, 
visa focalizar um conjunto de fatores baseado em duas categorias:

1) Processo Seletivo: será utilizado o desempenho do estudante no Exame Nacional do Ensino Médio (ENEM), que divide o conhecimento do estudante com base em 5 áreas, a saber: Ciências humanas e suas tecnologias; Linguagens, códigos e suas tecnologias; Ciências da natureza e suas tecnologias; Matemática e suas tecnologias; e Redação

2) Disciplinas Correlatas: serão utilizadas as disciplinas do primeiro semestre do curso de Ciência da Computação da Universidade Federal de Alagoas, a saber: Laboratório de Programação; Internet e Web; Fundamentos de Matemática; Geometria Analítica; e Inglês Instrumental ${ }^{2}$.

Nesse contexto, o objetivo do presente estudo pode ser especificado e, consequentemente, estratificado utilizando a abordagem de Goal, Question e Metrics (GQM) [Solingen and Berghout 1999]. Assim, focaliza-se o objetivo desse estudo, tal como segue:

O) Analisar o desempenho no processo seletivo e nas disciplinas correlatas no intuito de caracterizar um conjunto de correlações com respeito ao desempenho em Programação 1 no contexto de estudantes ingressantes no curso de Ciência da Computação da Universidade Federal de Alagoas nos anos de 2011 e 2012.

Com base nesse objetivo, é possível definir uma questão de pesquisa primária que irá nortear todo o estudo. Essa questão pode ainda derivar um conjunto de questionamentos secundários, que se mostram laterais ao presente estudo, mas que poderão futuramente ser foco de outros estudos. Desse modo, sumariza-se o estudo com base nas questões a seguir:

$\left.Q_{1}\right)$ Como o desempenho no processo seletivo e nas disciplinas correlatas se correlacionam com o desempenho de um estudante iniciante de programação?

$Q_{2}$ ) Como o desempenho nas áreas do processo seletivo se correlacionam entre si?

$Q_{3}$ ) Como o desempenho nas disciplinas correlatas se correlacionam entre si?

Visando responder os questionamentos apresentados, será utilizada uma métrica para mensurar o coeficiente de correlação. Em suma, diz-se que duas variáveis estão correlacionadas quando uma variação em uma das variáveis é geralmente acompanhada de uma variação na outra. Assim, duas variáveis podem apresentar uma correlação direta, ou seja, ambas variam positivamente, ou então uma correlação inversa, ou seja, uma variável varia positivamente e a outra negativamente. Formalmente, o coeficiente de correlação pode ser definido tal como segue:

\footnotetext{
${ }^{1}$ mais informações sobre o ENEM podem ser encontrada em http://www.enem.inep.gov.br/

${ }^{2}$ mais informações sobre as disciplinas podem ser encontradas em http://www.ufal.edu.br/
} 
$\rho: X_{1} \times X_{2} \rightarrow[-1,1]$, onde $\begin{cases}0.0=|\rho| & \text { nada se pode afirmar } \\ 0.0<|\rho| \leq 0.3 & \text { indica correlação fraca } \\ 0.3<|\rho| \leq 0.7 & \text { indica correlação moderada } \\ 0.7<|\rho| \leq 1.0 & \text { indica correlação forte }\end{cases}$

\subsection{Design e Execução do Estudo}

Tendo em vista os objetivos identificados anteriormente, é preciso primeiramente definir o conjunto de fatores que irão fazer parte do estudo. Nessa perspectiva, é possível definir tais fatores com base em três classes distintas:

a) Processo Seletivo: apresenta um conjunto composto pelos seguintes fatores: $\alpha_{1}$ ) Ciências humanas e suas tecnologias; $\alpha_{2}$ ) Linguagens, códigos e suas tecnologias; $\alpha_{3}$ ) Ciências da natureza e suas tecnologias; $\alpha_{4}$ ) Matemática e suas tecnologias; e $\alpha_{5}$ ) Redação. O desempenho é representado por uma variável quantitativa que assume valores no intervalo [400, 1000];

$\beta$ ) Disciplinas Correlatas: apresenta um conjunto composto pelos seguintes fatores: $\beta_{1}$ ) Laboratório de Programação; $\beta_{2}$ ) Introdução à Computação; $\beta_{3}$ ) Internet e Web; $\beta_{4}$ ) Fundamentos de Matemática; $\beta_{5}$ ) Geometria Analítica; e $\beta_{6}$ ) Inglês Instrumental. $\mathrm{O}$ desempenho é representado por uma variável quantitativa que assume valores no intervalo $[0,10]$;

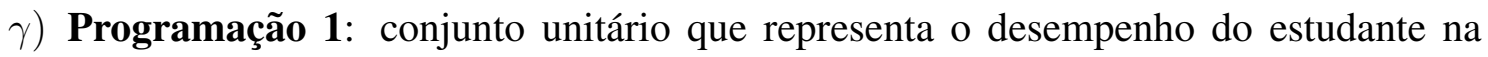
disciplina introdutória de programação, é representada por uma variável quantitativa que assume valores no intervalo $[0,10]$

Dando continuidade, a construção e execução do estudo pode ser definido com base em três etapas distintas:

1) Seleção dos fatores: remete diretamente aos objetivos apresentados anteriormente, ou seja, os fatores dependem diretamente do questionamento em foco. Nesse sentido, define-se um subconjunto de experimentos para cada um dos questionamentos levantados e, desse modo, cada questionamento acarreta na escolha dos seguintes fatores:

$\left.Q_{1}\right) \rho: \gamma \times \alpha_{i}$ e $\rho: \gamma \times \beta_{j}$ com $1 \leq i \leq 6 \wedge 1 \leq j \leq 5$

$\left.Q_{2}\right) \rho: \alpha_{i} \times \alpha_{j}$, com $1 \leq i \leq 5 \wedge 1 \leq j \leq 5 \wedge i \neq j$;

$\left.Q_{3}\right) \rho: \beta_{i} \times \beta_{j}$, com $1 \leq i \leq 6 \wedge 1 \leq j \leq 6 \wedge i \neq j$.

2) Seleção dos valores observados: foi definida com base na aplicação de um conjunto de filtros de exclusão definidos a seguir:

$\left.F_{1}\right)$ Reprovação por falta;

$\left.F_{2}\right)$ Aproveitamento de estudo;

$F_{3}$ ) Trancamento de disciplina;

$F_{4}$ ) Desempenho igual a 0 (zero), sendo considerado desistência. 
Durante a aplicação dos filtros e a consequente seleção dos valores observados foram tomadas algumas decisões ad hoc que seguem:

$D_{1}$ ) A disciplina de Geometria Analítica apresentou um número excessivo de observações a serem descartadas diminuindo drasticamente o tamanho do conjunto de observações. O motivo identificado foi uma taxa fora do normal no número de desistentes e de trancamentos de matrícula acarretado por diferenças ideológicas entre discentes e docente. Assim, resolveu-se não considerar a disciplina de Geometria Analítica para esse estudo;

$D_{2}$ ) A disciplina de Inglês Instrumental apresentou um número excessivo de observações a serem descartadas diminuindo drasticamente o tamanho do conjunto de observações. O motivo identificado foi um problema institucional que acarretou na ausência de professor por um grande período de tempo. Assim, resolveu-se não considerar a disciplina de Inglês Instrumental para esse estudo;

$D_{3}$ ) As observações do ano de 2011 não apresentavam desempenho no processo seletivo, haja vista que o processo conhecido como SISU começou a ser adotado na instituição apenas a partir do ano de 2012. Assim, optou-se por utilizar as observações do ano de 2011 apenas quando forem verificadas correlações entre disciplinas correlatas.

Após a seleção dos valores observados foram obtidos 47 observações para a turma do ano de 2011 e 61 observações para a turma do ano de 2012, totalizando 108 observações.

3) Execução do estudo: para a análise dos dados e aplicação dos testes estatísticos foram utilizados os seguintes instrumentos a seguir:

- Ambiente: R versão 3.0 .1 (2013-05-16)

- Sistema: Windows 8 (64 bits)

- Dataset: Computacao.csv ${ }^{3}$

- Execução: Run.R ${ }^{4}$

Após a escolha dos valores observados será verificada a normalidade dos dados e, após isso, poderá ser aplicado o teste de correlação de Pearson, caso a distribuição dos dados seja normal, ou então o teste de correlação de Spearman, caso a distribuição não seja normal.

\section{Resultados e Discussão}

Na seção em questão serão apresentados os resultados obtidos durante o estudo. Além disso, será inserida uma breve discussão visando, primeiramente, responder os questionamentos levantados inicialmente e, em seguida, apresentar novos desdobramentos. Assim, após a execução do estudo, foram obtidos os resultados apresentados na Figura 4 e Figura 4. Sob essa perspectiva, considerando os resultados obtidos é possível iniciar uma

\footnotetext{
${ }^{3}$ disponível em http://goo.gl/JLzAdm

${ }^{4}$ disponível em http://goo.gl/JLzAdm
} 
discussão no intuito de responder a cada um dos questionamentos levantados anteriormente:

$\left.Q_{1}\right)$ Como o desempenho no processo seletivo e nas disciplinas correlatas se correlacionam com o desempenho de um estudante iniciante de programação?

Com relação ao processo seletivo, a primeira linha da matriz da Figura 4 apresenta os resultados das correlações entre a disciplina de Programação 1 e as áreas de conhecimento do ENEM. Nesse sentido, há que se destacar:

- a correlação entre o desempenho em Programação 1 e o desempenho em Humanas, que apresentou uma correlação negativa moderada $(\rho=-0.38$, $p$ - valor < 0.010), ou seja, com base nos dados analisados a medida que o desempenho nesta área aumenta o desempenho em Programação decresce de um pouco;

- a correlação entre o desempenho em Programação 1 e o desempenho em Linguagens, que apresentou uma correlação negativa fraca $(\rho=-0.28$, $p$ - valor $<0.100$ ), ou seja, analogamente ao caso anterior existe uma correlação inversa, embora fraca, entre os dois fatores.

Com relação às disciplinas correlatas, a primeira linha da matriz da Figura 4 apresenta os resultados das correlações entre a disciplina de Programação 1 e as demais disciplinas. Nesse sentido, há que se destacar:

- a correlação entre o desempenho em Programação 1 e o desempenho em Laboratório de Programação, que apresentou uma correlação moderada ( $\rho=0.61$, $p$ - valor < 0.001). Nesse caso parece óbvio haver uma correlação entre essas duas disciplinas, haja vista que são disciplinas complementares, entretanto é interessante notar que embora o bom desempenho em Programação 1 implique um bom desempenho na disciplina de Laboratório, a recíproca não se mostra verdadeira. Nesse sentido, é possivel verificar pelo gráfico de dispersão apresentado na célula $(2,1)$ da Figura 4 , que existe um grande número de estudantes que obtiveram bom desempenho na disciplina de Laboratório, mas que não obtiveram bom desempenho em Programação 1;

- as correlações entre o desempenho em Programação 1 e o desempenho nas disciplinas de Internet e Web, Introdução à Computação e Fundamentos de Matemática, que apresentaram correlações positivas entre moderada e fraca (respectivamente, $\rho=0.43, p-$ valor $<0.001 ; \rho=0.37, p-$ valor $<0.001$; e $\rho=0.25, p-$ valor $<0.010)$.

$Q_{2}$ ) Como o desempenho nas áreas do processo seletivo se correlacionam entre si?

Com relação ao processo seletivo, as demais linhas da matriz da Figura 4 apresentam os resultados dessas correlações. Nesse sentido, há que se destacar: 


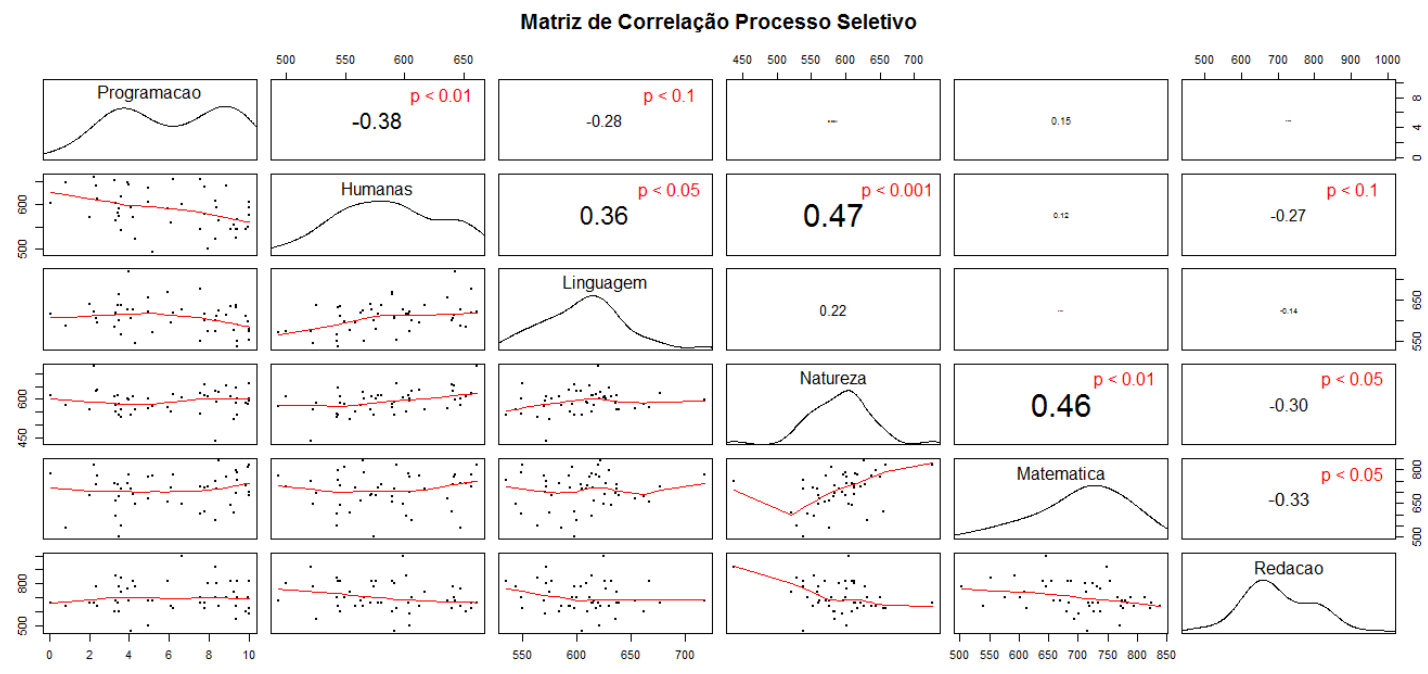

Figura 1. Matriz dos resultados para o processo seletivo: 1) a diagonal principal apresenta a distribuição dos fatores em foco; 2) cada célula $i, j$ abaixo da diagonal principal apresenta o gráfico de dispersão entre o fator da linha $i$ e o fator da coluna $j$; $\mathbf{3}$ ) cada célula $i, j$ acima da diagonal principal apresenta o $\boldsymbol{p}$-valor e o coeficiente de correlação entre o fator da linha $i$ e o fator da coluna $j$.

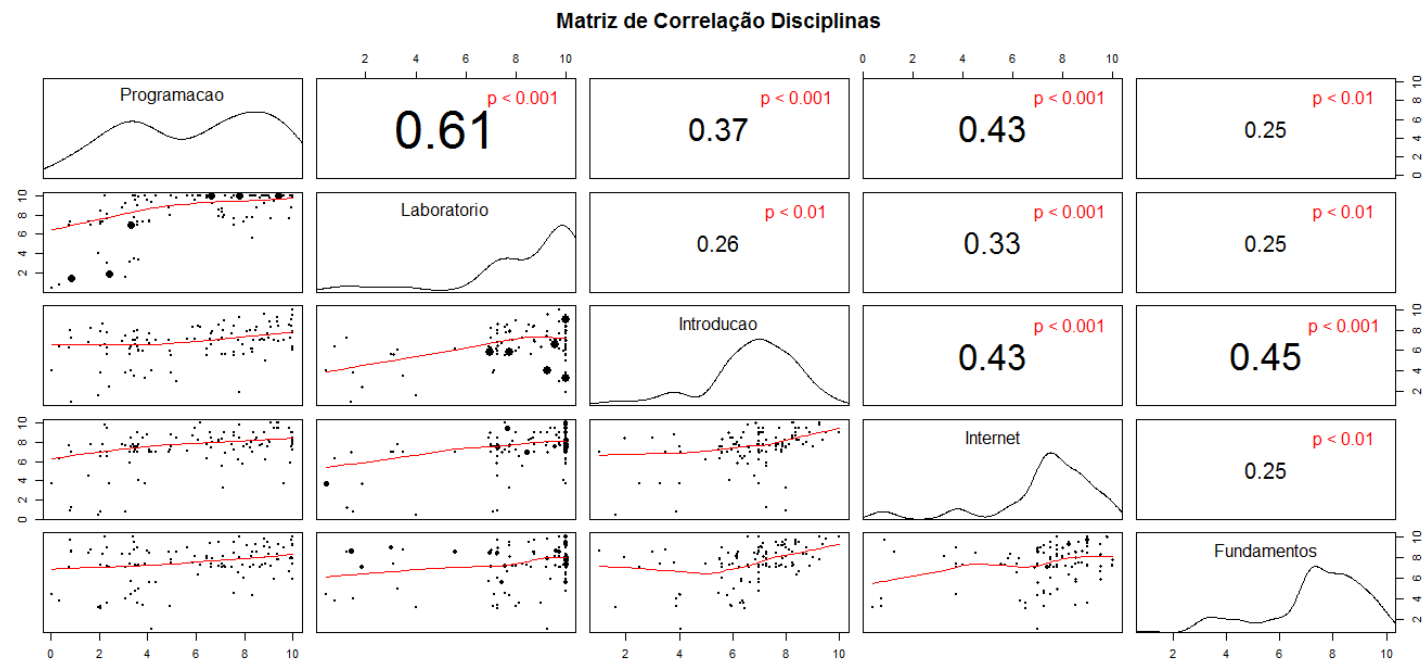

Figura 2. Matriz dos resultados para as disciplinas correlatas: 1) a diagonal principal apresenta a distribuição dos fatores em foco; 2) cada célula $i, j$ abaixo da diagonal principal apresenta o gráfico de dispersão entre o fator da linha $i$ e o fator da coluna $j$; 3) cada célula $i, j$ acima da diagonal principal apresenta o $\boldsymbol{p}$-valor e o coeficiente de correlação entre o fator da linha $i$ e o fator da coluna $j$. 
- as correlações entre o desempenho na área de Natureza e o desempenho nas áreas de Humanas e Matemática, que apresentaram correlações positivas moderadas (respectivamente, $\rho=0.47, p-$ valor $<0.001$; e $\rho=0.46$, $p-$ valor $<0.010$ ).

$Q_{3}$ ) Como o desempenho nas disciplinas correlatas se correlacionam entre si?

Com relação às disciplinas correlatas, as demais linhas da matriz da Figura 4 apresentam os resultados dessas correlações. Nesse sentido, há que se destacar:

- as correlações entre o desempenho na disciplina de Introdução à Computação e as disciplinas de Internet e Web e Fundamentos de Matemática, que apresentaram correlações positivas moderadas (respectivamente, $\rho=0.43, p-$ valor $<0.001$; $\rho=0.45, p-$ valor $<0.001)$.

De modo geral, as disciplinas correlatas se mostraram mais correlacionadas ao desempenho em Programação 1 do que o desempenho no processo seletivo. Nesse sentido, é importante destacar que este estudo ainda é inicial e que foi utilizado apenas o ano de 2012 para mensurar as correlações com o processo seletivo. Outro ponto a ser destacado foi a fraca correlação entre o desempenho de Programação 1 e o desempenho na disciplina de Fundamentos de Matemática e a área de Matemática do ENEM, pois esperava-se que houvesse um coeficiente de correlação maior. Assim, espera-se realizar novos experimentos com dados do ano de 2013 e, provavelmente, com dados de outras instituições a fim de verificar novamente estas correlações.

\section{Considerações Finais}

O presente trabalho apresentou um estudo que visou identificar correlações entre o desempenho em programação e o desempenho tanto no processo de seleção quanto nas disciplinas correlatas de um curso de Ciência da Computação da Universidade Federal de Alagoas. Nesse sentido, foram utilizados dados das turmas dos anos de 2011 e 2012 e foram verificadas correlações com a métrica de Spearman.

Dentre os resultados obtidos, é importante ressaltar um coeficiente de correlação moderado encontrado entre o desempenho em Programação 1 e a disciplina de Laboratório de Programação $(\rho=0,61, p$ - valor $<0.001)$, ressaltando-se, no entanto, que não foi encontrada uma relação bidirecional entre essas duas disciplinas, ou seja, nem todo bom desempenho na disciplina de Laboratório acarretou em bom desempenho na disciplina de Programação 1. Outro resultado encontrado foi a correlação inversa entre a disciplina de Programação 1 e as áreas de Humanas e Linguagens do processo de seleção (respectivamente, $\rho=-0.38, p-$ valor $<0.010$; e $\rho=0.28, p-$ valor $<0.100$ ). Além disso, esperava-se um coeficiente de correlação maior entre a disciplina de Programação 1 e a disciplina de Fundamentos de Matemática, bem como da própria área de matemática do exame seletivo, todavia, para ambos os casos não foram encontrados correlações fortes.

Por fim, acredita-se que esse estudo tenha contribuído como mais um trabalho que visa caracterizar o problema que é o ensino de programação. Nesse sentido, acredita-se que ainda há muito a fazer e, como tal, acredita-se que o entendimento desse problema em sua plenitude remete a não menos do que uma abordagem tanto quantitativa, que foi iniciada 
nesse trabalho, como qualitativa. Assim, definem-se como direcionamentos futuros a análise de novos dados, para uma abordagem quantitativa, e a aplicação de entrevista tanto com professores quanto com alunos, análise de fóruns de discussão e um levantamento bibliográfico sistemático, no intuito de caracterizar o problema do ensino de programação.

\section{Referências}

Bennedsen, J. and Caspersen, M. E. (2007). Failure rates in introductory programming. SIGCSE Bull., 39(2):32-36.

Bergin, S. and Reilly, R. (2005). Programming: factors that influence success. In Proceedings of the 36th ACM technical symposium on Computer science education, SIGCSE '05, pages 411-415, New York, NY, USA. ACM.

Carbone, A., Hurst, J., Mitchell, I., and Gunstone, D. (2009). An exploration of internal factors influencing student learning of programming. In Proceedings of the 11th Australasian Conference on Computing Education, ACE '09, pages 25-34, Darlinghurst, Australia, Australia. Australian Computer Society, Inc.

de Jesus, E. A. and Raabe, A. L. A. (2009). Interpretações da Taxonomia de Bloom no Contexto da Programação Introdutória. In Anais do $20^{\circ}$ Simpósio Brasileiro de Informática na Educação, volume 20 of SBIE'09. Sociedade Brasileira de Computação.

Hertz, M. and Ford, S. M. (2013). Investigating factors of student learning in introductory courses. In Proceeding of the 44th ACM technical symposium on Computer science education, SIGCSE '13, pages 195-200, New York, NY, USA. ACM.

Lahtinen, E., Ala-Mutka, K., and Järvinen, H.-M. (2005). A study of the difficulties of novice programmers. In Proceedings of the 10th ACM conference on Innovation and technology in computer science education, ITiCSE '05, pages 14-18, New York, NY, USA. ACM.

McGettrick, A., Boyle, R., Ibbett, R., Lloyd, J., Lovegrove, G., and Mander, K. (2005). Grand challenges in computing: Education-a summary. Computer Journal, 48(1):4248.

Pears, A., Seidman, S., Malmi, L., Mannila, L., Adams, E., Bennedsen, J., Devlin, M., and Paterson, J. (2007). A survey of literature on the teaching of introductory programming. In Working group reports on ITiCSE on Innovation and technology in computer science education, ITiCSE-WGR '07, pages 204-223, New York, NY, USA. ACM.

Piva Jr, D. and Freitas, R. L. (2010). Estratégias para melhorar os processos de abstração na disciplina de Algoritmos. In Anais do $21^{\circ}$ Simpósio Brasileiro de Informática na Educação, volume 21 of SBIE'10. Sociedade Brasileira de Computação.

Powers, K., Gross, P., Cooper, S., McNally, M., Goldman, K. J., Proulx, V., and Carlisle, M. (2006). Tools for teaching introductory programming: what works? In Proceedings of the 37th ACM technical symposium on Computer science education, SIGCSE '06, pages 560-561, New York, NY, USA. ACM.

Solingen, R. V. and Berghout, E. (1999). Goal/Question/Metric Method: A Practical Guide for Quality Improvement of Software Development. McGraw-Hill Inc. 